\title{
Kapellasite: A Kagome Quantum Spin Liquid with Competing Interactions
}

\author{
B. Fåk, ${ }^{1}$ E. Kermarrec,${ }^{2}$ L. Messio, ${ }^{3}$ B. Bernu,${ }^{4}$ C. Lhuillier, ${ }^{4}$ F. Bert, ${ }^{2}$ P. Mendels,${ }^{2,5}$ B. Koteswararao, ${ }^{2}$ \\ F. Bouquet, ${ }^{2}$ J. Ollivier, ${ }^{6}$ A. D. Hillier, ${ }^{7}$ A. Amato, ${ }^{8}$ R. H. Colman, ${ }^{9}$ and A. S. Wills ${ }^{9}$ \\ ${ }^{1}$ SPSMS, UMR-E CEA/UJF-Grenoble-1, INAC, F-38054 Grenoble Cedex 9, France \\ ${ }^{2}$ Laboratoire de Physique des Solides, Université Paris Sud 11, UMR CNRS 8502, F-91405 Orsay, France \\ ${ }^{3}$ Institute of Theoretical Physics, École Polytechnique Fédérale de Lausanne, CH-1015 Lausanne, Switzerland \\ ${ }^{4}$ LPTMC, UMR 7600 CNRS, Université Pierre et Marie Curie, Paris VI, F-75252 Paris Cedex 05, France \\ ${ }^{5}$ Institut Universitaire de France, 103 bd Saint-Michel, F-75005 Paris, France \\ ${ }^{6}$ Institut Laue Langevin, BP156, F-38042 Grenoble, France \\ ${ }^{7}$ ISIS Facility, STFC, Rutherford Appleton Laboratory, Chilton, Didcot, Oxon OX11 OQX, United Kingdom \\ ${ }^{8}$ Laboratory for Muon Spin Spectroscopy, Paul Scherrer Institut, CH-5232 Villigen PSI, Switzerland \\ ${ }^{9}$ University College London, Department of Chemistry, 20 Gordon Street, London, WClH OAJ, United Kingdom
}

(Received 23 March 2012; revised manuscript received 9 May 2012; published 20 July 2012)

\begin{abstract}
Magnetic susceptibility, NMR, muon spin relaxation, and inelastic neutron scattering measurements show that kapellasite, $\mathrm{Cu}_{3} \mathrm{Zn}(\mathrm{OH})_{6} \mathrm{Cl}_{2}$, a geometrically frustrated spin-1/2 kagome antiferromagnet polymorphic with herbertsmithite, is a gapless spin liquid showing unusual dynamic short-range correlations of noncoplanar cuboc 2 type which persist down to $20 \mathrm{mK}$. The Hamiltonian is determined from a fit of a high-temperature series expansion to bulk susceptibility data and possesses competing exchange interactions. The magnetic specific heat calculated from these exchange couplings is in good agreement with experiment. The temperature dependence of the magnetic structure factor and the muon relaxation rate are calculated in a Schwinger-boson approach and compared to experimental results.
\end{abstract}

DOI: 10.1103/PhysRevLett.109.037208

The quantum spin liquid (QSL) is one of the most elusive states in condensed matter physics, even after two decades of intense studies [1], with implications ranging from geometrically or exchange frustrated magnetic systems to unconventional superconductivity. A promising model system for the observation of the QSL state is the $S=1 / 2$ kagome antiferromagnet (KAFM), which is characterized by quantum spins on a geometrically frustrated lattice of corner-sharing equilateral triangles. The first suitable model material, the mineral herbertsmithite, is classified as a gapless spin liquid [2-8] and still lacks a complete understanding. Its discovery triggered a burst of experimental and theoretical investigations in the field of quantum spin liquids in two dimensions and challenges the most advanced theoretical approaches [9-12].

A new experimental realization of the $S=1 / 2 \mathrm{KAFM}$ model was recently synthesized [13,14], the mineral kapellasite, $\mathrm{Cu}_{3} \mathrm{Zn}(\mathrm{OH})_{6} \mathrm{Cl}_{2}$, a polymorph of herbertsmithite; i.e., they have the same chemical formula but different crystallographic structures. The layered structure of kapellasite prevents the $\mathrm{Cu} / \mathrm{Zn}$ disorder present in these materials from either coupling the kagome planes or creating free spins, in contrast to the more three-dimensional herbertsmithite. In this Letter, we use inelastic neutron scattering and muon spin relaxation ( $\mu \mathrm{SR})$ down to $20 \mathrm{mK}$ to show that kapellasite is a gapless spin liquid without any sign of long-range magnetic order or spin freezing. In contrast to the featureless excitation spectrum of herbertsmithite, kapellasite exhibits novel dynamic short-range correlations with a well-defined wave-vector dependence
PACS numbers: 75.10.Kt, 75.10.Jm, 75.40.-s, 78.70.Nx

(see Fig. 1), reminiscent of the noncoplanar twelvesublattice cuboc2 magnetic structure [15], where, due to a particular set of ferro- and antiferromagnetic competing interactions, spins on a given hexagon are coplanar with neighboring spins forming an angle of $60^{\circ}$ with each other; the third-neighbor spins across hexagons are antiparallel.

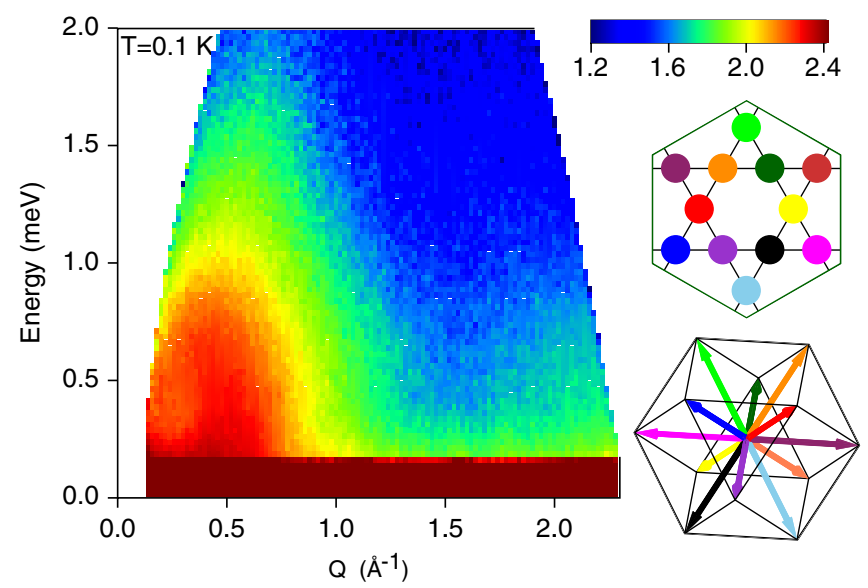

FIG. 1 (color online). Neutron scattering intensity $S(Q, E)$ on a logarithmic scale as a function of wave vector $Q$ and energy $E$ of kapellasite at a temperature of $0.1 \mathrm{~K}$ measured with an incoming neutron energy of $3.27 \mathrm{meV}$. The diffuse inelastic magnetic scattering is centered at $Q=0.5$ and $2.0 \AA^{-1}$. On the right are shown the spin directions (bottom) on the kagome lattice (middle) of the cuboc 2 magnetic structure. 
(a)

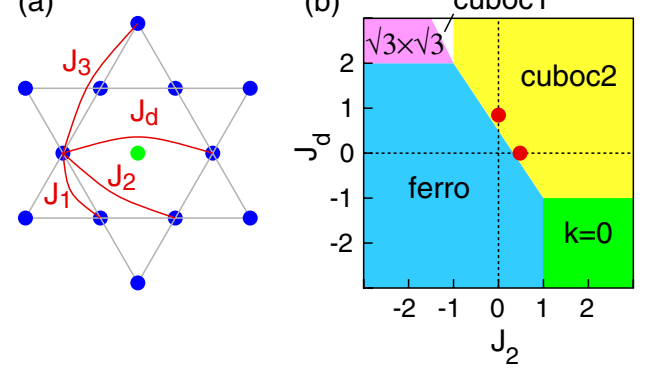

FIG. 2 (color online). (a) Kagome plane of kapellasite with $\mathrm{Cu}^{2+} S=1 / 2$ spins (blue), nonmagnetic $\mathrm{Zn}^{2+}$ ion (green), and exchange interactions (red). (b) Classical $J_{2}-J_{d}$ phase diagram for ferromagnetic nearest-neighbor coupling, $J_{1}=-1$, and $J_{3}=0$. The best two-parameter HT-series fits to susceptibility data are shown with red points.

Kapellasite crystallizes in the $P \overline{3} m 1$ trigonal space group (No. 164) with lattice parameters $a=6.33$ and $c=5.70 \AA$. The $S=1 / 2$ kagome lattice of undistorted corner-sharing triangles is obtained by regularly doping a two-dimensional (2D) triangular $\mathrm{Cu}^{2+}$ metal-site sublattice with diamagnetic $\mathrm{Zn}^{2+}$ ions [see Fig. 2(a)]. Inductively coupled plasma mass spectrometry showed that our sample has not the ideal $\mathrm{Cu} / \mathrm{Zn}$ 3:1 stoichiometry and ${ }^{35} \mathrm{Cl} \mathrm{NMR}$ showed some $\mathrm{Cu} / \mathrm{Zn}$ intersite mixing [16]. The actual chemical formula consistently determined with neutron powder diffraction [14] is $\left(\mathrm{Cu}_{0.73} \mathrm{Zn}_{0.27}\right)_{3}\left(\mathrm{Zn}_{0.88} \mathrm{Cu}_{0.12}\right) \times$ $(\mathrm{OH})_{6} \mathrm{Cl}_{2}$, with $27 \% \mathrm{Zn}$ on the $\mathrm{Cu}$-sites of the kagome lattice and $12 \% \mathrm{Cu}$ on the "hexagonal" $\mathrm{Zn}$ site. The $\mathrm{Cu} / \mathrm{Zn}$ mixing leads to some disorder within the kagome planes but cannot induce any coupling between the planes, which occurs only via very weak $\mathrm{O}-\mathrm{H}-\mathrm{Cl}$ hydrogen bonds [13]. Kapellasite is therefore remarkably two-dimensional.

The dc magnetic susceptibility measured in a SQUID in a magnetic field of $5 \mathrm{~T}$ exhibits a Curie-Weiss behavior $\chi_{\mathrm{dc}}(T)=C /\left(T-\theta_{\mathrm{CW}}\right)$ above $\sim 50 \mathrm{~K}$ with a weak ferromagnetic effective coupling, $\theta_{\mathrm{CW}}=9.5 \pm 1 \mathrm{~K}$ [Fig. 3(a)]. For $T \lesssim 50 \mathrm{~K}$, the susceptibility becomes Curie-like, with neither any sign of a magnetic transition nor a FC/ZFC opening. A similar Curie tail is observed in many other compounds and is often attributed to a defect contribution that exceeds the intrinsic susceptibility [5,17]. The use of a local probe such as NMR is decisive in this context: We find that the local susceptibility $\chi_{\text {loc }}$, extracted from the ${ }^{35} \mathrm{Cl}$ NMR shift of an oriented powder sample with magnetic fields up to $13 \mathrm{~T}$ applied perpendicular to the kagome planes, scales with $\chi_{\mathrm{dc}}$ over the whole temperature range $2-287 \mathrm{~K}$. This clearly shows that isolated spin defects are not present in kapellasite in contrast with the behavior seen in herbertsmithite, where $\mathrm{Cu} / \mathrm{Zn}$ mixing creates paramagnetic-like spins. Thus, the intrinsic susceptibility of kapellasite displays genuine divergent behavior pointing to a gapless spin state, rather than the downturn seen in other KAFM compounds.

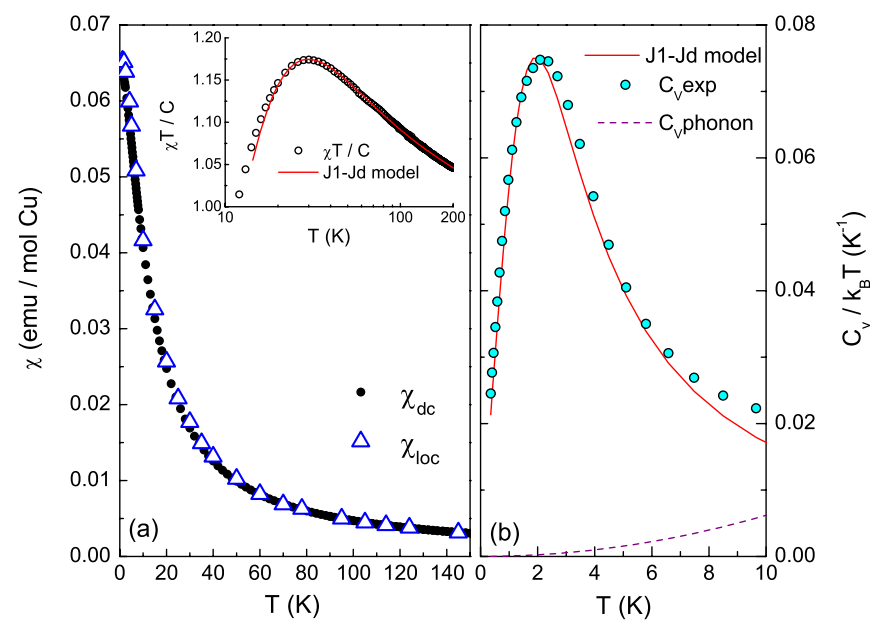

FIG. 3 (color online). (a) Local $\chi_{\mathrm{loc}}$ and macroscopic $\chi_{\mathrm{dc}}$ magnetic susceptibilities from NMR and dc SQUID measurements, respectively. Inset: Fit of high-temperature series expansion (red line) down to $20 \mathrm{~K}$. (b) Total specific heat per spin $C_{\mathrm{V}}^{\exp }$ measured in zero field (symbols), magnetic specific heat calculated with the $J_{1}-J_{d}$ model (red solid line) rescaled by a factor of 0.88 to account for mass uncertainties and missing entropy. The phonon contribution estimated from a high- $T$ fit (purple dashed line) is negligible below $10 \mathrm{~K}$.

A reasonable first theoretical description of kapellasite, which is deep in the Mott phase with well-defined localized spins, is the Heisenberg Hamiltonian

$$
\mathcal{H}=\sum_{\langle i, j\rangle_{\alpha}} J_{\alpha} \mathbf{S}_{i} \cdot \mathbf{S}_{j},
$$

where the exchange integrals $J_{\alpha}$ are defined in Fig. 2(a). We use $n$th order high-temperature (HT) series expansions $[18,19]$ to fit bulk $\chi_{\mathrm{dc}}$ data using $J_{1}-J_{2}$ or $J_{1}-J_{d}$ models, following suggestions that $J_{d}$ is significant in kapellasite [20]. In both cases, $J_{1}$ comes out ferromagnetic in competition with further-neighbor antiferromagnetic interactions [see red points in Fig. 2(b)], which explains the low value of $\theta_{\mathrm{CW}}$. Since a $n$th order HT series expansion depends on combinations of up to $n$th points correlation functions that describe the short-range arrangements of the spins, it is not surprising that the two fits fall in the same phase. The fits are very stable, and moving the parameters from the cuboc 2 phase towards the ferromagnetic one, say, leads rapidly to a poor agreement between theory and experiment. The fit of the $J_{1}-J_{d}$ model $(n=11)$ is qualitatively better than the $J_{1}-J_{2}$ model and gives a nearest-neighbor interaction $J_{1}=-15.0(4) \mathrm{K}$ and an "across-hexagon" interaction $J_{d}=12.7(3) \mathrm{K}$. The ferromagnetic nearest-neighbor exchange $\left(J_{1}\right)$ highlights the difference between kapellasite and its polymorph herbertsmithite and can be traced back to the $\mathrm{Cu}-\mu_{3} \mathrm{OH}-\mathrm{Cu}$ bonding angle being $\sim 13^{\circ}$ smaller in kapellasite [14,21]. The low- $T$ peak in the specific heat is also well captured by a HT-series expansion using the above $J_{1}-J_{d}$ parameters, as shown in Fig. 3(b). 
Inelastic neutron scattering (INS) measurements were performed at $0.1<T<300 \mathrm{~K}$ on the neutron time-offlight spectrometer IN5 at the ILL, using incident neutron energies of $0.82,3.27$, and $20.45 \mathrm{meV}$. A $93 \%$ deuterated powder sample of mass $3 \mathrm{~g}$ was held in an annular aluminium can and thermalized by helium exchange gas. Standard data reduction [22] gave the neutron scattering function $S(Q, E)$, shown in Fig. 1. The magnetic scattering, which dominates the nuclear (phonon) scattering for the energies and wave vectors studied in this work, is quasielastic and essentially without dispersion.

The inelastic magnetic structure factor $S_{\text {inel }}^{\mathrm{mag}}(Q)$, obtained by integrating $S(Q, E)$ over the energy range $0.4<$ $E<0.8 \mathrm{meV}$ and correcting for the magnetic form factor of the $\mathrm{Cu}^{2+}$ ions, is shown in Fig. 4(a). The measured $S_{\text {inel }}^{\mathrm{mag}}(Q)$ has initially only a weak temperature dependence but appears to shift to slightly lower $Q$ values for $T>15 \mathrm{~K}$. At even higher temperatures the structure gradually disappears, but short-range correlations persist up to at least $100 \mathrm{~K}$. At low temperatures, the broad peak near $Q=0.5 \AA^{-1}$ [see Fig. 4(a)] is centered at a much smaller value than in other kagome systems. The observed peak position corresponds neither to the $\Gamma$ point $(\mathbf{k}=0)$ in the Brillouin zone nor to the $K$ point $\mathbf{k}=(1 / 3,1 / 3)$ of the $\sqrt{3} \times \sqrt{3}$ structure but is close to the $M$ point, $\mathbf{k}=(1 / 2,0)$. The $M$ point on the kagome lattice corresponds to a unique spin arrangement among all possible regular spin states [23] of the Hamiltonian (1), called cuboc2 (see inset of Fig. 1 and Ref. [24]), and is fully consistent with the analysis of the HT spin susceptibility and the classical phase diagram of the derived Hamiltonian

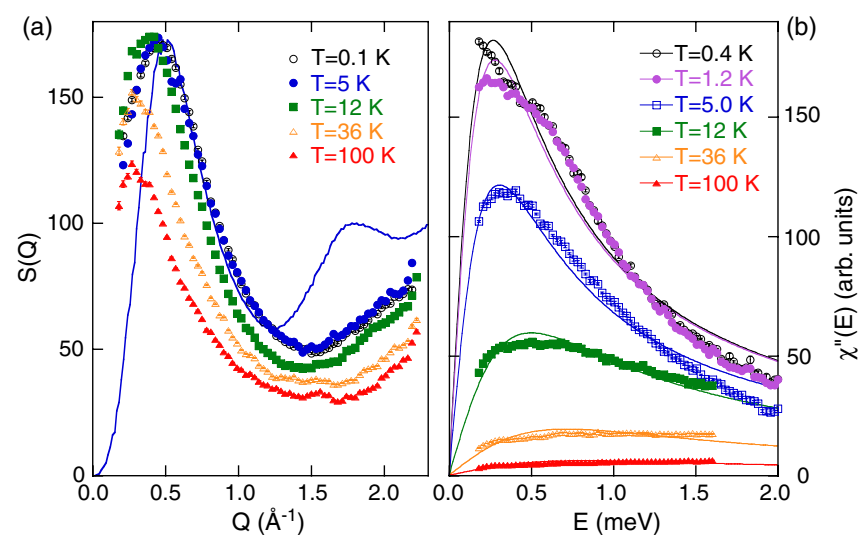

FIG. 4 (color online). (a) Wave-vector dependence of the inelastic magnetic scattering $S_{\text {inel }}^{\mathrm{mag}}(Q)$ integrated over the energy range $0.4<E<0.8 \mathrm{meV}$. Symbols are experimental data for different temperatures (statistical errors are smaller than the symbol size) and the line is the theoretical SBMF calculation at $T=5 \mathrm{~K}$. (b) Energy dependence of the imaginary part of the magnetic dynamic susceptibility $\chi^{\prime \prime}(E)$ integrated over the $Q$ range $0.4<Q<0.8 \AA^{-1}$ for different temperatures. The energy resolution is $0.11 \mathrm{meV}$. The data show deviations from a quasielastic Lorentzian (lines) at low temperatures. [see Fig. 2(b)]. These two independent measurements, in the high- and low-temperature regimes, give strong support that the short-range correlations of kapellasite are of noncoplanar cuboc 2 type.

To confirm the relevance of the cuboc 2 state for quantum spins, we have explored the impact of quantum fluctuations on the classical system by employing a Schwinger-boson mean-field approach (SBMF) [25] of the Hamiltonian (1), using the values of $J_{1}$ and $J_{d}$ extracted from $\chi_{\mathrm{dc}}$ and a spin value of $S=0.5$. The powder-averaged structure factor $S(Q)$ calculated at $T=5 \mathrm{~K}$ for the same energy range as in the experiment is displayed as a line in Fig. 4(a), with only the overall scale factor adjusted. The position of the first peak in $S(Q)$ at $Q=0.5 \AA^{-1}$ is in good agreement with experiment, as is the weak intensity near $Q=1 \AA^{-1}$. These two features are the main signatures of the cuboc2 phase. Classical simulations show that replacing $\mathrm{Cu}$ by $\mathrm{Zn}$ on $27 \%$ of the kagome sites broaden the structure factor so that the peaks at large $Q$ s are partly blurred out while the first peak in the powder-averaged $S(Q)$ near $Q=0.5 \AA^{-1}$ appears at slightly smaller $Q$ s [26]. Adding quantum fluctuations to this dilution effect could lead to even better agreement with INS data.

The spin dynamics studied by INS displays a smooth continuum of excitations up to at least $2 \mathrm{meV}$ with no discernible gap and no sign of damped spin waves, see Fig. 1. We extract the energy dependence by integrating $S(Q, E)$ over the wave vector range $0.4<Q<0.8 \AA^{-1}$, where the magnetic scattering is strong and the nuclear scattering negligible, and divide by the temperature factor $1-\exp \left(-E / k_{B} T\right)$ to obtain the imaginary part of the magnetic dynamic susceptibility, $\chi^{\prime \prime}(E)$, shown in Fig. 4(b). At high temperatures, $\chi^{\prime \prime}(E)$ is well described by a quasielastic Lorentzian $\chi^{\prime \prime}(E)=\chi^{\prime} E \Gamma /\left(E^{2}+\Gamma^{2}\right)$ similar to the classical $(S=5 / 2)$ KAFM deuteronium jarosite [27]. For $T>5 \mathrm{~K}$, the line width (inverse relaxation rate) increases as $\Gamma(T) \propto T^{1 / 3}$ with temperature while the staggered static susceptibility decreases as $\chi^{\prime}(T) \propto$ $T^{-2 / 3}$. At lower temperatures, below $5 \mathrm{~K}$, the shape of $\chi^{\prime \prime}(E)$ deviates from a single Lorentzian, which signals the onset of quantum spin-liquid correlations. However, kapellasite does not show the $E^{-\alpha}$ behavior of pyrochlore slabs [28] nor the $E / T$ scaling of herbertsmithite [4,29]. This gives further support for kapellasite not being close to a quantum critical point. A full understanding of the spinliquid dynamics of kapellasite is not yet at hand and will be discussed further below.

Further insight into the low- $T$ state of kapellasite is provided by our $\mu$ SR experiments, which were performed on a nonoriented powder sample in zero field (ZF) and longitudinal applied field (LF) at the ISIS and PSI facilities down to $20 \mathrm{mK}$. The evolution of the muon polarization is plotted in Fig. 5(a), both in zero field and under $H=$ $100 \mathrm{G}$ from $4 \mathrm{~K}$ to $20 \mathrm{mK}$. The relaxation of the muon polarization can be fitted in the whole $T$ range to the sum of 


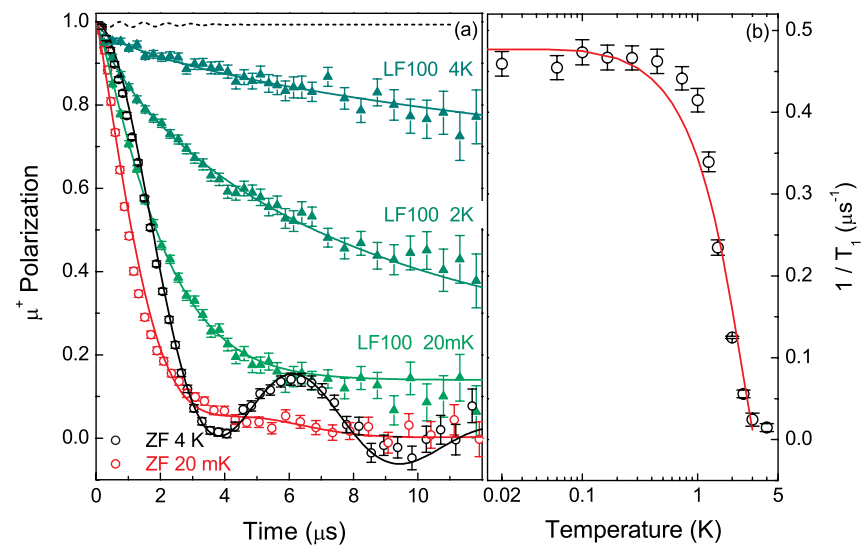

FIG. 5 (color online). (a) Time dependent muon polarization in a zero field (open symbols) and a longitudinal field (closed symbols) demonstrate the absence of spin freezing. Solid lines are fits to Eq. (2) while the dashed line represents the expected $\mathrm{LF}$ polarization at $100 \mathrm{G}$ for frozen spins at $20 \mathrm{mK}$. (b) Temperature dependence of the relaxation rate $1 / T_{1}$ from $\mu \mathrm{SR}$ measurements (circles) and SBMF calculations (red line).

the local responses of two muon sites, known to be located nearby $\mathrm{OH}^{-}$and $\mathrm{Cl}^{-}[3]$,

$$
P(t)=f P_{\mathrm{OH}}(t) e^{-\lambda_{\mathrm{OH}} t}+(1-f) K_{\mathrm{Cl}}(t) e^{-\lambda_{\mathrm{Cl}} t},
$$

where $f \sim 0.86$ is the $T$-independent fraction of muons on the $\mathrm{OH}^{-}$site and $\lambda_{\mathrm{OH} / \mathrm{Cl}}$ is the dynamical electronic relaxation rate probed at the $\mathrm{OH}^{-} / \mathrm{Cl}^{-}$site. Both $P_{\mathrm{OH}}(t)$ and the Kubo-Toyabe function $K_{\mathrm{Cl}}(t)$ are related to the nuclear static fields [3] that are $T$ independent and were fixed to their $T=4 \mathrm{~K}$ values.

Any spin freezing can be ruled out based on the following arguments. First, in a fully static scenario, the low- $T$ relaxation should be ascribed to a distribution of static internal fields with a width $\Delta H \sim 6 \mathrm{G}$ that should be completely decoupled in an applied LF of $100 \mathrm{G}$. The dashed line in Fig. 5(a) shows that this is clearly not the case. Second, the ZF polarization at $20 \mathrm{mK}$ relaxes to zero, not showing the long-time 1/3-component characteristic of frozen moments. Finally, the $T$ dependence of the ZF polarization is well described by Eq. (2) in the whole temperature range from $20 \mathrm{~K}$ to $20 \mathrm{mK}$ with only one adjustable parameter $\lambda_{\mathrm{OH}}$ to account for the $\mathrm{Cu}$ spin dynamics. No electronic relaxation $\left(e^{-\lambda_{\mathrm{Cl}} t} \sim 1\right)$ was found at the $\mathrm{Cl}$ site, which is further away from the magnetic planes.

To study the slow spin dynamics, an applied field of $100 \mathrm{G}$ along the $\mu^{+}$polarization decouples the static nuclear magnetism so that Eq. (2) becomes $P(t)=f e^{-\lambda t}+$ $(1-f)$, and the spin-lattice relaxation rate $1 / T_{1}=\lambda_{\mathrm{OH}}$ is probed directly [Fig. 5(b)]. Above $4 \mathrm{~K}$, the $S=1 / 2$ kagome spins are in a rapidly fluctuating regime. From $3 \mathrm{~K}$ to $0.8 \mathrm{~K}$ we observe a slowing down of spin fluctuations, which occurs in the same $T$ range as the change in dynamics in neutron experiments and coincides with the low- $T$ specific heat peak. This indicates an enhancement of the short-range correlations at low $T$, and the corresponding energy scale is quite low as a result from a competition between the exchange interactions. Finally, persistent fluctuations are observed down to $20 \mathrm{mK}$. This behavior points to a gapless spin-liquid state with an upper bound of a hypothetical spin gap of $20 \mathrm{mK}$, i.e., $10^{-3} J_{1}$. Within our SBMF approach, the $\mu$ SR spin-lattice relaxation rate can be calculated as $1 / T_{1} \propto$ $T\left[\chi^{\prime \prime}\left(\omega_{L}, T\right) / \omega_{L}\right]$, where $\omega_{L}$ is the muon Larmor frequency. The theoretical results, also shown in Fig. 5(b), are in excellent qualitative agreement with the experiment, demonstrating that the local dynamics of kapellasite is well captured by the SBMF calculations at low frequencies. We note that the SBMF calculations for both the muon relaxation rate and $S(Q, E)$ give essentially the same predictions forboth the $J_{1}-J_{d}$ and $J_{1}-J_{2}$ models. This suggests that the importance lies not in the exact values of the exchange parameters used, but rather in which part of the phase diagram kapellasite is located, and this is unambiguously determined by the HT expansion.

At higher frequencies, our SBMF calculations predict well-defined spin waves for $T<3 \mathrm{~K}$ with energies around $0.5 \mathrm{meV}$. We expect that dilution of the magnetic sites reduces the spin-spin correlation length and broadens the modes. However, $S(Q, E)$ would still display reminiscences of spin waves, resulting in a nonmonotonous energy response for certain $Q$ values (such as the magnetic zone boundary), which is clearly not the case in kapellasite, where the energy dependence of the intensity at all $Q$ s decreases monotonically. It thus appears clear that our a priori choice of bosonic excitations is too restrictive to capture both the continuum of spinon excitations and the absence of a gap. This indeed calls for further theoretical explorations. A fermionic chiral approach would plausibly lead both to gapless and diffuse excitations and to a better description of the inelastic neutron spectrum. Zerotemperature fermionic approaches of the pure antiferromagnetic Heisenberg Hamiltonian [9,30,31] have been extremely interesting and similar work should be extended to the present model with competing interactions, with the aim to describe both chiral physics and thermodynamic properties. Whether the dilution of $\mathrm{Cu}$ ions is strong enough to kill the predicted low- $T$ chiral phase expected with this local spin arrangement [32] is also an interesting question.

This work was supported in part by the European Commission under the 6th Framework Programme Contract No. RII3-CT-2003-505925, ANR Grant No. ANR-09-JCJC-0093-01 and ARCUS Ile de FranceInde. We thank C. Baines for assistance at PSI, D. Dragoe for the ICP analysis, and P. Viot and F. Brieuc for their collaboration on the simulation of disorder.

[1] L. Balents, Nature (London) 464, 199 (2010).

[2] M.P. Shores, E. A. Nytko, B. M. Bartlett, and D. G. Nocera, J. Am. Chem. Soc. 127, 13462 (2005). 
[3] P. Mendels, F. Bert, M. A. de Vries, A. Olariu, A. Harrison, F. Duc, J.C. Trombe, J.S. Lord, A. Amato, and C. Baines, Phys. Rev. Lett. 98, 077204 (2007).

[4] J. S. Helton et al., Phys. Rev. Lett. 98, 107204 (2007).

[5] A. Olariu, P. Mendels, F. Bert, F. Duc, J. C. Trombe, M. A. de Vries, and A. Harrison, Phys. Rev. Lett. 100, 087202 (2008).

[6] M. A. de Vries, J. R. Stewart, P. P. Deen, J. O. Piatek, G. J. Nilsen, H. M. Rønnow, and A. Harrison, Phys. Rev. Lett. 103, 237201 (2009).

[7] P. Mendels and F. Bert, J. Phys. Soc. Jpn. 79, 011001 (2010).

[8] M. Jeong, F. Bert, P. Mendels, F. Duc, J. C. Trombe, M. A. de Vries, and A. Harrison, Phys. Rev. Lett. 107, 237201 (2011).

[9] Y. Ran, M. Hermele, P. A. Lee, and X. G. Wen, Phys. Rev. Lett. 98, 117205 (2007).

[10] S. Yan, D. A. Huse, and S. R. White, Science 332, 1173 (2011).

[11] Y.-M. Lu, Y. Ran, and P. A. Lee, Phys. Rev. B 83, 224413 (2011).

[12] L. Messio, B. Bernu, and C. Lhuillier, Phys. Rev. Lett. 108, 207204 (2012).

[13] R. H. Colman, C. Ritter, and A. S. Wills, Chem. Mater. 20, 6897 (2008).

[14] R. H. Colman, A. Sinclair, and A. S. Wills, Chem. Mater. 22, 5774 (2010).

[15] J.-C. Domenge, P. Sindzingre, C. Lhuillier, and L. Pierre, Phys. Rev. B 72, 024433 (2005).

[16] E. Kermarrec et al. (to be published)

[17] J. A. Quilliam, F. Bert, R. H. Colman, D. Boldrin, A.S. Wills, and P. Mendels, Phys. Rev. B 84, 180401(R) (2011).
[18] B. Bernu and G. Misguich, Phys. Rev. B 63, 134409 (2001).

[19] G. Misguich and B. Bernu, Phys. Rev. B 71, 014417 (2005).

[20] O. Janson, J. Richter, and H. Rosner, Phys. Rev. Lett. 101, 106403 (2008).

[21] A. S. Wills and J.-Y. Henry, J. Phys. Condens. Matter 20, 472206 (2008).

[22] LAMP, http://www.ill.eu/data_treat/lamp/the-lamp-book/.

[23] L. Messio, C. Lhuillier, and G. Misguich, Phys. Rev. B 83, 184401 (2011).

[24] See Supplemental Material at http://link.aps.org/ supplemental/10.1103/PhysRevLett.109.037208 for a three-dimensional animation of the cuboc 2 structure.

[25] L. Messio, O. Cépas, and C. Lhuillier, Phys. Rev. B 81, 064428 (2010).

[26] See Supplemental Material at http://link.aps.org/ supplemental/10.1103/PhysRevLett.109.037208 for details on the classical simulation.

[27] B. Fåk, F. C. Coomer, A. Harrison, D. Visser, and M. E. Zhitomirsky, Europhys. Lett. 81, 17006 (2008).

[28] C. Broholm, G. Aeppli, G. P. Espinosa, and A. S. Cooper, Phys. Rev. Lett. 65, 3173 (1990).

[29] J. S. Helton, K. Matan, M. P. Shores, E. A. Nytko, B. M. Bartlett, Y. Qiu, D. G. Nocera, and Y. S. Lee, Phys. Rev. Lett. 104, 147201 (2010).

[30] Y. Iqbal, F. Becca, and D. Poilblanc, Phys. Rev. B 83, 100404(R) (2011).

[31] Y. Iqbal, F. Becca, and D. Poilblanc, Phys. Rev. B 84, 020407(R) (2011).

[32] J.-C. Domenge, C. Lhuillier, L. Messio, L. Pierre, and P. Viot, Phys. Rev. B 77, 172413 (2008). 